\title{
A CHARACTERIZATION OF CONTINUA THAT CONTAIN NO $n$-ODS AND NO $W$-SETS
}

\author{
ELDON J. VOUGHT
}

(Communicated by Dennis Burke)

\begin{abstract}
A proper, nondegenerate subcontinuum $K$ of a continuum $Y$ is said to be a $W$-set if, for every continuum $X$ and map $f$ of $X$ onto $Y$, some subcontinuum of $X$ is mapped by $f$ onto $K$. Jim Davis asked whether a simple closed curve is the only atriodic continuum that contains no $W$-set. An affirmative answer is given to this question. The result follows as a corollary to the more general theorem that a continuum contains no $n$-od and has no $W$-set if and only if it is a graph in which every point is contained in a simple closed curve. Properties of this class of graphs are also described.
\end{abstract}

Van Nall [3, p. 192] has proved that a decomposable continuum is a simple closed curve if and only if it is atriodic and contains no $W$-sets. In this paper, "decomposable" is removed from the hypothesis by proving that an atriodic, indecomposable continuum contains a $W$-set. This result follows from part of the proof of the main theorem that characterizes a continuum that contains no $n$-ods, for some integer $n$, and has no $W$-sets as a graph in which every point is contained in a simple closed curve.

\section{DEFINITIONS AND PRELIMINARIES}

A continuum is a compact, connected metric space. A proper, nondegenerate subcontinuum $K$ of a continuum $Y$ is a $W$-set in $Y$ if, for every continuum $X$ and map $f$ of $X$ onto $Y$, some subcontinuum of $X$ is mapped by $f$ onto $K$. A continuum belongs in class $W$ if every proper, nondegenerate subcontinuum is a $W$-set. A graph is a continuum that is the union of a finite number of arcs and contains only a finite number of points of order different from 2 . A continuum $L$ is an $n-o d, n \geq 2$, if $L$ contains a subcontinuum $H$, called the $h u b$, whose complement has at least $n$ components. A continuum $X$ is called a $\theta_{n}$-continuum, $n \geq 1$, if no subcontinuum of $X$ separates it into more

Received by the editors May 15, 1989 and, in revised form, August 7, 1989. The results of this paper were presented at the Spring Topology Conference on April 17, 1989 at the University of Tennessee, Knoxville, Tennessee.

1980 Mathematics Subject Classification (1985 Revision). Primary 54F20; Secondary 54B15, $54 \mathrm{C} 05$.

Key words and phrases. Continuum, $W$-set, $n$-od, atriodic, class $W, \theta_{n}$-continuum, graph, monotone upper-semicontinuous decomposition, quotient space. 
than $n$ components. Note that a continuum that contains no $n$-ods must be a $\theta_{n-1}$-continuum. Also, if $x \in X$ and $H_{1}$ and $H_{2}$ are disjoint sets in $X$, $x$ cuts $H_{1}$ from $H_{2}$ if every subcontinuum of $X$ that intersects $H_{1}$ and $H_{2}$ contains $x$.

Suppose $f$ is a map from a continuum $X$ onto a continuum $Y, H$ is a closed subset of $Y$, and $y \in Y \backslash H$. Let $U_{1}, U_{2}, \ldots$ be a nested sequence of open sets in $X$ such that $f^{-1}(H)=\bigcap_{i=1}^{\infty} U_{i}$ where $U_{1} \cap f^{-1}(y)=\phi$, and let $x$ be an element of $f^{-1}(y)$. For each $i$, let $C_{i}$ be the component of $X \backslash U_{i}$ that contains $x$. For each $i$, let $C_{y, H}^{i}=f\left(C_{i}\right)$ and observe that $C_{y, H}^{i} \subset C_{y, H}^{j}$ if $i<j$. Let $C_{y, H}=\operatorname{cl}\left(\bigcup_{i=1}^{\infty} C_{y, H}^{i}\right)=f\left(\operatorname{cl}\left(\bigcup_{i=1}^{\infty} C_{i}\right)\right)$, and note that $y \in C_{y, H}$, $H \cap C_{y, H} \neq \phi$, and $C_{y, H}$ is the image of a subcontinuum of $X$ (namely $\left.\operatorname{cl}\left(\bigcup_{i=1}^{\infty} C_{i}\right)\right)$. In addition, $C_{y, H}$ is not unique since it depends on the choice of $x$ in $f^{-1}(y)$.

The following theorem, a corollary of [4, Theorem 2, p. 635], is needed.

Theorem A. Let $Y$ be a hereditarily decomposable $\theta_{n}$-continuum. Then $Y$ admits a monotone, upper-semicontinuous decomposition $\mathscr{D}$, the elements of which have void interior and which is unique and minimal with respect to the property that the quotient space $Y / D$ is a graph.

Each element of the decomposition is called a tranche, the order of a tranche is the order of the tranche considered as a point in the quotient space. If $D_{1}$ and $D_{2}$ are two tranches in $\mathscr{D}$, a connected subset $C$ of $Y \backslash\left(D_{1} \cup D_{2}\right)$ that is the union of tranches and has limit points in $D_{1}$ and $D_{2}$ is called a segment between $D_{1}$ and $D_{2}$, if all tranches in $C$ are of order 2 (note that $D_{1}$ or $D_{2}$ may not be of order 2). Then $D_{1}$ and $D_{2}$ are called end tranches of the segment and the usual real number notation $\left[D_{1}, D_{2}\right],\left[D_{1}, D_{2}\right),\left(D_{1}, D_{2}\right],\left(D_{1}, D_{2}\right)$, specifies whether $D_{1}$ and $D_{2}$ is to be included in the segment.

\section{Proof of THE MAIN THEOREM}

We start with several lemmas that lead to a proof of the main theorem.

Lemma 1. If $Y$ is a continuum that contains no $W$-sets and for some integer $n \geq 3$ has no $n$-ods, then every proper, nondegenerate subcontinuum of $Y$ is decomposable.

Proof. Suppose $Y$ contains a proper, indecomposable subcontinuum $I$, and let $f$ be a map from a continuum $X$ onto $Y$ such that no subcontinuum of $X$ maps onto $I$. For each natural number $k$, let $P_{k}$ be the statement that $Y$ contains mutually disjoint subcontinua, $H_{1}, \ldots, H_{k}$ such that for each $i$, $1 \leq i \leq k, H_{i} \backslash I \neq \phi, H_{i} \cap I \neq \phi$, and $I \backslash \bigcup_{i=1}^{k} H_{i} \neq \phi$. Choose points $z, w$ in different composants of $I$ and consider the continuum $C_{z,\{w\}}$. Since no subcontinuum of $X$ maps onto $I$ and $\{z, w\} \subset C_{z,\{w\}}, C_{z,\{w\}} \not \subset I$. There exists an integer $i$ such that $C_{z,\{w\}}^{i} \not \subset I$. But $w \notin C_{z,\{w\}}^{i}$. Hence it follows 
that $H_{1}=C_{z,\{w\}}^{i}$ and $I$ are as required in $P_{1}$. Suppose $P_{k}$ is true, and let $H_{1}, \ldots, H_{k}$ be subcontinua as required in $P_{k}$. Assume for some $i, 1 \leq i \leq k$, $H_{i} \cap I$ is not connected, and let $H_{i} \cap I=A \cup B$, a separation. There exist open sets $U$ and $V$ (open relative to $H_{i}$ ) with disjoint closures such that $A \subset U$ and $B \subset V$. By [2, Theorem 50, p. 18], there exist continua $A_{1}, B_{1}$ in $H_{i}$, such that $A_{1} \subset \mathrm{cl}(U), B_{1} \subset \mathrm{cl}(V), A_{1} \cap A \neq \phi \neq A_{1} \cap \mathrm{bd}(U)$, and $B_{1} \cap B \neq \phi \neq$ $B_{1} \cap \operatorname{bd}(V)$. Then $\left\{H_{j}: 1 \leq j \leq k, j \neq i\right\} \cup\left\{A_{1}, B_{1}\right\}$ are $k+1$ subcontinua of $Y$ as required in $P_{k+1}$. Assume that for each $i, 1 \leq i \leq k, H_{i} \cap I$ is connected, let $H=\bigcup_{i=1}^{k} H_{i}$, and choose $z$ in a different composant of $I$ from the composants that contain $\left\{H_{i} \cap I: 1 \leq i \leq k\right\}$. Since no subcontinuum of $X$ maps onto $I$ and $C_{z, H}$ contains $z$ and intersects $H, C_{z, H} \not \subset I$. There exists an integer $i$ such that $C_{z, H}^{i} \not \subset I$. But $C_{z, H}^{i} \cap H=\phi$, so it follows that $C_{z, H}^{i}$ is a subcontinuum $H_{k+1}$ that together with $H_{1}, \ldots, H_{k}$ are the required continua in $P_{k+1}$. By induction, $P_{n}$ is true. However, $I \cup\left(\bigcup_{i=1}^{n} H_{i}\right)$ is an $n$-od, a contradiction, so the lemma is true.

Lemma 2. If $Y$ is a continuum that contains no $W$-sets and, for some integer $n \geq 3$, has no $n$-ods, then every proper subcontinuum of $Y$ is locally connected.

Proof. By Lemma 1, each proper, nondegenerate subcontinuum $W$ of $Y$ is decomposable. By hypothesis, $Y$ (and hence $W$ ) contains no $n$-ods, and $W$ is therefore a $\theta_{n-1}$-continuum. Theorem A guarantees that $W$ admits a unique monotone, upper-semicontinuous decomposition $\mathscr{D}$ for which the tranches have void interior and for which $W / \mathscr{D}$ is a graph. To show local connectivity, it suffices to prove that each tranche in $\mathscr{D}$ is degenerate. Suppose $T$ is a nondegenerate tranche in $\mathscr{D}$. Then there exists a segment $\left[T, T_{1}\right]$ and a nondegenerate subcontinuum $T^{\prime}$ of $T$ such that $T^{\prime}=T \cap \operatorname{cl}\left(\left(T, T_{1}\right]\right)$. Let $X$ be a continuum and $f$ a map of $X$ onto $Y$ such that no subcontinuum of $X$ maps onto $T^{\prime}$. Suppose for each tranche $T^{*}$ in $\left(T, T_{1}\right]$ there exists a continuum $H^{*}$ in $X$ such that $f\left(H^{*}\right) \cap\left(T, T^{*}\right) \neq \phi$ and $f\left(H^{*}\right) \cap\left(Y \backslash\left[T, T^{*}\right]\right) \neq \phi$, but $f\left(H^{*}\right) \cap\left(T \cup T^{*}\right)=\phi$. Then there exist $n+1$ tranches, $T_{1}, \ldots, T_{n+1}$, and $n$ subcontinua of $X, H_{1}, \ldots, H_{n}$ such that $T<T_{n+1}<\cdots<T_{1}$, and for $1 \leq i \leq n, f\left(H_{i}\right) \cap\left(T_{i+1}, T_{i}\right) \neq \phi, f\left(H_{i}\right) \cap Y \backslash\left[T_{i+1}, T_{i}\right] \neq \phi$, but $f\left(H_{i}\right) \cap\left(T_{i} \cup T_{i+1}\right)=\phi$. Then $\left[T, T_{1}\right] \cup \bigcup_{i=1}^{n} f\left(H_{i}\right)$ contains an $n$-od, a contradiction.

Hence, there exists a tranche (assume without loss of generality that it is $\left.T_{1}\right)$ such that for each $y$ in $\left(T, T_{1}\right)$ and each $i, C_{y, K}^{i} \subset\left(T, T_{1}\right)$ where $K=T_{1} \cup T$. Then $C_{y, K} \subset\left[T, T_{1}\right]$ and either $C_{y, K} \cap T_{1} \neq \phi$ or $C_{y, K} \cap T^{\prime} \neq \phi$. There exists a continuum $H$ in $X$ such that $f(H) \subset\left[T, T_{1}\right]$ and $f(H) \cap T^{\prime} \neq$ $\phi \neq f(H) \cap\left(T, T_{1}\right]$. To see this, note that it follows immediately if $C_{y, K} \cap T \neq \phi$ for some $y$ in $\left(T, T_{1}\right)$. So assume that $C_{y, K} \cap T_{1} \neq \phi$ and $C_{y, K} \cap T^{\prime}=\phi$ for each $y$ in $\left(T, T_{1}\right)$. Then by taking a suitable limit $H$ of the continua in $X$ that map onto the $C_{y, K}, H$ is the required continuum. So $f \mid H$ is a map from 
$H$ into $\left[T, T_{1}\right]$ such that the image of $H$ intersects $\left(T, T_{1}\right]$ and contains $T^{\prime}$, since $\operatorname{cl}\left(T, T_{1}\right)$ is irreducible between $T^{\prime}=T \cap \operatorname{cl}\left(T, T_{1}\right)$ and $T_{1} \cap \operatorname{cl}\left(T, T_{1}\right)$ [1, Lemma 1, p. 262].

Now for each $y$ in $f(H)$ and each closed subset $K$ of $f(H) \backslash\{y\}$, let us consider the sets $C_{y, K}$ relative to $f \mid H$. Fix a point $z$ in $T^{\prime}$, and consider any other point $y$ in $T^{\prime}$. For each $i, C_{z,\{y\}}^{i}$ contains $z$ but not $y$, so $C_{z,\{y\}}^{i} \subset T^{\prime}$ [1, Lemma 1, p. 262]. Therefore, $C_{z,\{y\}} \subset T^{\prime}$. Then if the same point $x$ in $f^{-1}(z) \cap H$ is used to define $C_{z,\{y\}}$ for each $y$ in $T^{\prime} \backslash\{z\}$, then $T^{\prime}=\{z\} \cup\left\{C_{z,\{y\}}: y \in T^{\prime} \backslash\{z\}\right\}$ will be the image of a subcontinuum of $H$ under $f$. But $T^{\prime}$ was assumed to not be the image of any subcontinuum in $X$ under $f$. This contradiction establishes the lemma.

Lemma 3. If $Y$ is a continuum that contains no $W$-sets and, for some integer $n \geq 3$, has no n-ods, then $Y$ is decomposable.

Proof. Suppose $Y$ is indecomposable. It follows from Lemma 2 that each composant is arcwise connected. If the order of a point in $y$ in $Y$ is defined as the maximum integer $k$ such that there is a simple $k$-od in $Y$ with hub $y$, then it follows that each composant contains at most a finite number of points whose order is different from 2. For, if not, it would be possible to construct a $k$-od for arbitrarily large $k$. Choose a composant $C$, and let $K$ be a subcontinuum of $C$ irreducible about the points in $C$ of order different from 2 (if there are no points of order different from 2, let $K$ be any singleton set). Let $\mathscr{D}$ be the monotone, upper-semicontinuous decomposition of $Y$ whose only nondegenerate element is $K$ (unless $K$ is degenerate, in which case $\mathscr{D}$ is the set of singletons). Clearly $Y^{\prime}=Y / \mathscr{D}$ is an indecomposable continuum. Denote $K$, as a point of $Y^{\prime}$, by $k^{\prime}$. Since $Y^{\prime}$, as well is $Y$, contains no $n$-ods, there is a ray $R$, i.e., $R$ is the continuous one-to-one image of $[0, \infty)$, in the composant of $Y^{\prime}$ containing $k^{\prime}$ whose only endpoint is $k^{\prime}$ and which is dense in $Y^{\prime}$. Choose $r$ in $R$ and an open set $U$ such that $r \in U$ and $k^{\prime} \notin \operatorname{cl}(U)$. Every nondegenerate subcontinuum contained in $\operatorname{cl}(U) \cap R$ is an arc since all the points in $C$ of order different from 2 are contained in $K$. Since $R$ is dense in $Y^{\prime}$, in $\operatorname{cl}(U) \cap R$ there exist mutually disjoint arcs $A, A_{1}, A_{2}, \ldots$ such that $r \in A, \lim A_{i}=A$, and $r$ is between $k^{\prime}$ and $A_{i}$ on $R$ for each $i$. For each $i$, let the endpoints of $A_{i}$ be $a_{i}$ and $b_{i}$ and let the endpoints of $A$ be $p$ and $q$. Because $A$ is not a $W$-set, there exist a continuum $X$ and a map $f$ from $X$ onto $Y$ such that no subcontinuum of $X$ maps onto $A$. Since either $p$ or $q$ cuts the other from $K$ in $C$, we can assume without loss of generality that $p$ cuts $q$ from $K$. For each $j$, either $a_{j}$ or $b_{j}$ cuts the other from $q$. Renaming if necessary, assume that $a_{j}$ cuts $b_{j}$ from $q$ for each $j$. Let $H_{j}=\left\{p, b_{j}\right\}$ for each $j$, and consider $C_{q, H_{j}}$ for a specific $j$. Since $C_{q, H_{j}}^{i}$ for each $i$ lies in the arc from $p$ to $b_{j}, C_{q, H_{j}}$ lies in the arc and contains either $p$ or $b_{j}$. If, for some $j, C_{q, H_{j}}$ contains $p$, then $C_{q, H_{j}}$ contains $A$. Since an arc is in class $W$ and there is 
a continuum in $X$ that maps onto the arc $C_{q, H_{j}}$, there exists a continuum in $X$ that maps onto $A$. But this is not possible, because no subcontinuum of $X$ maps onto $A$.

So for each $j, C_{q, H_{j}}$ contains $b_{j}$, and hence $A_{j}$, but not $p$. Hence there exists a continuum $T_{j}$ in $X$ such that $f\left(T_{j}\right)=A_{j}$. Choosing subsequences if necessary, let $T=\lim T_{j}$. Since $\lim A_{j}=A$, it follows that $f(T)=A$. Again, this is not possible, and the contradiction establishes the lemma.

The continuum $Y$ of Lemmas 1, 2, and 3, is hereditarily decomposable and every proper subcontinuum is locally connected. So $Y$ is a $\theta_{n-1}$-continuum that is locally connected since all its tranches are degenerate. Therefore, $Y$ is equivalent to its unique minimal monotone, upper-semicontinuous decomposition for which the quotient space is a graph. Hence $Y$ is a graph.

We now prove the main theorem of the paper.

Theorem 1. The continuum $Y$ contains no $W$-sets and, for some integer $n \geq 3$, has no $n$-ods if and only if $Y$ is a graph in which each point is contained in a simple closed curve.

Proof. To prove the necessity, we will use the fact that $Y$ is a graph. Suppose $y$ is a point of order 2 that is not contained in a simple closed curve. Then $y$ is a separating point of $Y$ since $Y$ is locally connected. Hence, there exists a segment $A$ containing $y$ such that $A$ separates $Y$. Let $Y \backslash A=P \cup Q$, a separation, and denote by $p$ and $q$ the endpoints of $A$ such that $\operatorname{cl}(P) \cap A=$ $\{p\}, \operatorname{cl}(Q) \cap A=\{q\}$. Because $A$ is not a $W$-set, there exist a continuum $X$ and a map $f$ from $X$ onto $Y$ such that no subcontinuum of $X$ maps onto $A$. Let $A_{p}$ be the set of all points $x$ in $f^{-1}(A)$ such that the component of $x$ in $f^{-1}(A)$ has a limit point in $f^{-1}(p)$. Define $A_{q}$ analogously. Then $f^{-1}(A)=A_{p} \cup A_{q}$ and, because $A$ is not a $W$-set, $\operatorname{cl}\left(A_{p}\right) \cap A_{q}=\phi=\operatorname{cl}\left(A_{q}\right) \cap A_{p}$; i.e., $A_{p}$ and $A_{q}$ are disjoint closed sets. Then $\left[f^{-1}(P) \cup A_{p}\right] \cup\left[f^{-1}(Q) \cup A_{q}\right]$ is a separation of $X$, a contradiction, and so every point of order 2 is contained in a simple closed curve. There are only a finite number of points in $Y$ of order different from 2. If $y$ is such a point, then there is a segment in $Y$ with one endpoint $y$. Then the simple closed curve that contains one of the points of the segment contains $y$. This completes the proof of the necessity.

For the sufficiency, let $Y$ be a graph such that each point is contained in a simple closed curve. A graph has only a finite number of points of order different from 2 , and each point of order $k$ different from 2 is the hub of a simple $k$-od (in fact, $k>2$ since there are no endpoints in $Y$ ). It follows that $Y$ contains no $n$-ods for some integer $n$ sufficiently large.

Next, suppose that $K$ is a proper nondegenerate subcontinuum of $Y$. Let $[a, b]$ be a segment that is properly contained in $K$, and let $x_{1}, x_{2}, z, y_{2}, y_{1}$ be points of $(a, b)$ in the order from $a$ to $b$. Choose $r$ in $Y \backslash K$. Define a function $f$ from $Y$ onto $Y$ which is the identity on $Y \backslash[a, b]$, and maps the following arcs homeomorphically: $a x_{1}$ onto an arc ar not containing $z, b y_{1}$ 
onto an arc $b r$ not containing $z, x_{1} x_{2}$ onto $\operatorname{rax}_{1} x_{2} z, y_{1} y_{2}$ onto $r b y_{1} y_{2} z$, $x_{2} z$ onto $z x_{2} x_{1} a r$, and $y_{2} z$ onto $z y_{2} y_{1} b r$. No subcontinuum of $Y$ maps onto $K$, and thus the proof is complete.

\section{AdDitional facts}

The next theorem discusses the nature of the graphs yielded by Theorem 1 .

Theorem 2. Let $Y$ be a graph with the property that every point is contained in a simple closed curve. If $n$ is an integer, $n \geq 2$, such that $Y$ contains an $n$-od but no $(n+1)$-od, then $n$ is even and $Y$ is the union of $n / 2$ simple closed curves such that no one curve is contained in the union of the others.

Proof. Let $K$ be an $n$-od in $Y$ with hub $H$. Then $K$ contains all the junction points of $Y$.

To see this, suppose that $y$ is a junction point of $Y$ that is not in $K$, and let $A$ be an arc in $Y$ from $K$ to $y$. There exist segments $B$ and $C$ such that $B \cap K=\phi, C \cap K=\phi$, and $A \cap B=A \cap C=B \cap C=\{y\}$. But $K \cup A \cup B \cup C$ contains an $(n+1)$-od, a contradiction. In fact, the hub $H$ contains all the junction points. To see this, assume that the junction point $y \notin H$. Then $y$ lies in $Q$, one of the $n$ connected sets that comprise $K \backslash H$. Let $A$ be an arc in $\operatorname{cl}(Q)$ from $y$ to $H$, and let $B$ and $C$ be segments such that $B \cap H=\phi$, $C \cap H=\phi$, and $A \cap B=A \cap C=B \cap C=\{y\}$. Then $(K \backslash Q) \cup A \cup B \cup C$ is an $(n+1)$-od with hub $H \cup A$, a contradiction.

The $n$-od $K$ contains no simple closed curve. Assume that it does. Then there exists an open segment $A$ in $K$ such that $K \backslash A$ is connected and is an $n$-od. Then $K$ is an $(n+1)$-od, a contradiction.

Since $K$ is an $n$-od, let $K=H \cup \bigcup_{i=1}^{n} Q_{i}$ where the $Q_{i}$ 's are mutually disjoint connected sets. Each component of $Y \backslash K$ is an open segment with endpoints in different $Q_{i}$ 's. Also, different components of $Y \backslash K$ cannot have endpoints in the same $Q_{i}$ or else $Y$ would contain an $(n+1)$-od. So $n$ is even and $Y \backslash K$ has $n / 2$ components. Each component of $Y \backslash K$ is contained in a simple closed curve; label these simple closed curves $S_{1}, \ldots, S_{n / 2}$. Then $\bigcup_{i=1}^{n / 2} S_{i}$ has the property that no $S_{i}$ is contained in the union of the others. Since $K$ contains no simple closed curve, $Y=\bigcup_{i=1}^{n / 2} S_{i}$, and the theorem is proved.

Note that $K$ in Theorem 2 is a finite tree, and if $\mathscr{D}$ is the monotone, uppersemicontinuous decomposition whose only nondegenerate element is $K$, then $Y / \mathscr{D}$ is a generalized figure- 8 curve with $n / 2$ loops.

Theorems 1 and 2 also yield the following corollary.

Corollary 1. If $Y$ is an atriodic continuum that contains no $W$-sets, then $Y$ is a simple closed curve and conversely.

Theorem 1 can be slightly strengthened as follows. If we define a proper, nondegenerate subcontinuum $K$ of $Y$ to be a $W^{\prime}$-set in $Y$ if for every map 
$f$ of $Y$ onto $Y$, some subcontinuum of $Y$ is mapped onto $K$, then a $W$ set is clearly a $W^{\prime}$-set. The following theorem is a slightly stronger version of Theorem 1.

Theorem 3. The continuum $Y$ contains no $W^{\prime}$-sets and for some integer $n \geq 3$ has no n-ods if and only if $Y$ is a graph in which each point is contained in a simple closed curve.

Proof. Clearly if $Y$ contains no $W^{\prime}$-sets, then $Y$ contains no $W$-sets, so the necessity follows from Theorem 1 . Since the function $f$ defined in the proof of sufficiency in Theorem 1 is a map from $Y$ onto $Y$, the sufficiency also follows from Theorem 1.

\section{REFERENCES}

1. E. E. Grace and Eldon J. Vought, Monotone decompositions of $\theta_{n}$-continua, Trans. Amer. Math. Soc. 263 (1981), 261-270.

2. R. L. Moore, Foundations of point set theory, Amer. Math. Soc. Colloq. Publ. 13 (1962).

3. V. C. Nall, Weak confluence and W-sets, Topology Proc. 8 (1983), 161-193.

4. Eldon J. Vought, $\omega$-connected continua and Jones' $K$ function, Proc. Amer. Math. Soc. 91 (1984), 633-636.

Department of Mathematics, California State University, Chico, California 959290525 